Supporting Information

\title{
Noninvasive Early Detection of Calpain 2-Enriched Non-Small Cell Lung Cancer Using a Human Serum Albumin-Bounded Calpain-2 Nanosensor
}

\author{
Seung-Hae Kwon ${ }^{l, *}$, Taemin Wi ${ }^{l, *}$,Yong Il Park ${ }^{2}$, Min Woo Kim³ ${ }^{3}$ Gibok Lee ${ }^{2}$, Takumi Higaki ${ }^{3}$, \\ Jung Hoon Choi ${ }^{4, *}$ and Ruda Lee $e^{3, *}$ \\ ${ }^{1}$ Korea Basic Science Institute, Seoul 02841, South Korea \\ ${ }^{2}$ School of Chemical Engineering, Chonnam National University, Gwangju 61186, South Korea \\ ${ }^{3}$ International Research Organization for Advanced Science and Technology, Kumamoto University, Kumamoto 860-8555, \\ Japan \\ ${ }^{4}$ Department of Anatomy \& Institute of Veterinary Science, College of Veterinary Medicine, Kangwon National University, \\ Chuncheon 24341, South Korea
}




\section{Materials and Methods}

\section{Analysis of tumor-targeting efficiency}

To estimate tumor-targeting efficiency, we measured the fluorescence signals in the whole body (yellow line) and tumor (red line) regions, respectively. The targeting efficiency was calculated using:

[Targeting efficiency $(\%)=$ fluorescence signal in tumor area / fluorescence signal in whole body area*100]

\section{Results and Discussion}

The HSA-CAPN2 showed $8.16 \%$ of the fluorescence signal in tumors. Compare with HSA-CAPN2, HSA-Cy5 and CAPN2 showed $1.1 \%$ and $1.6 \%$ of fluorescence signals at tumor sites, respectively (Figure S5). HSA-CAPN2 showed fluorescence signals in the liver and intestine as well as in the tumor. However, the activated fluorescence signal in the tumor showed the highest intensity among other fluorescence regions. Although tumor accumulation is not that much compared to the liver and intestine, HSA-CAPN2 has been shown to increase targeting capacity. 
A

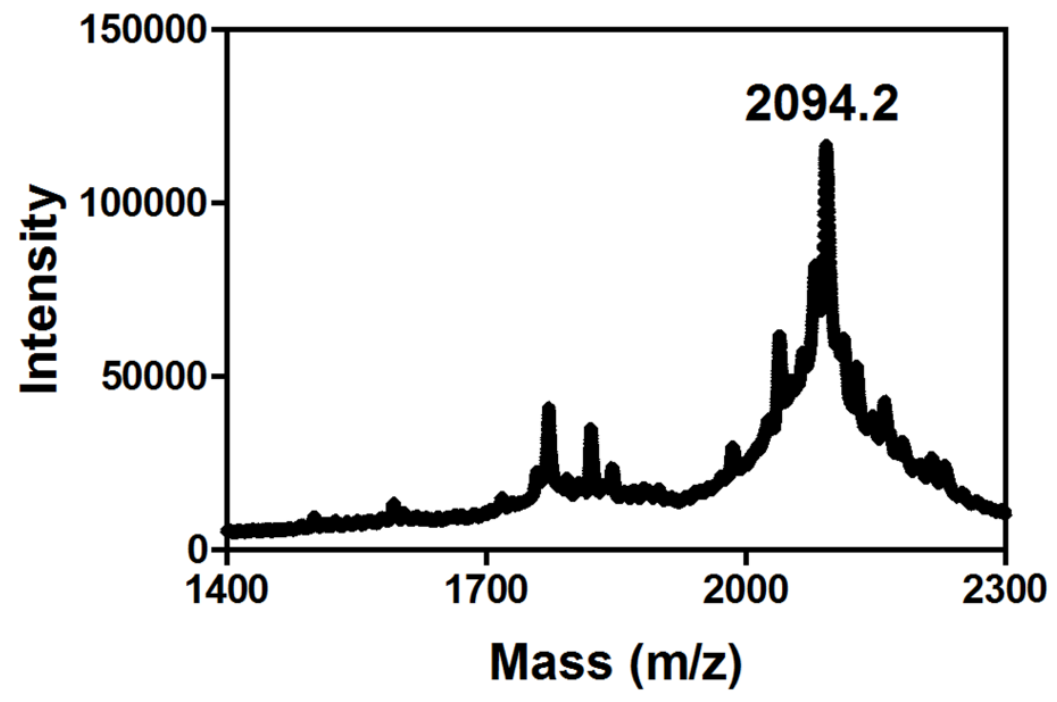

B

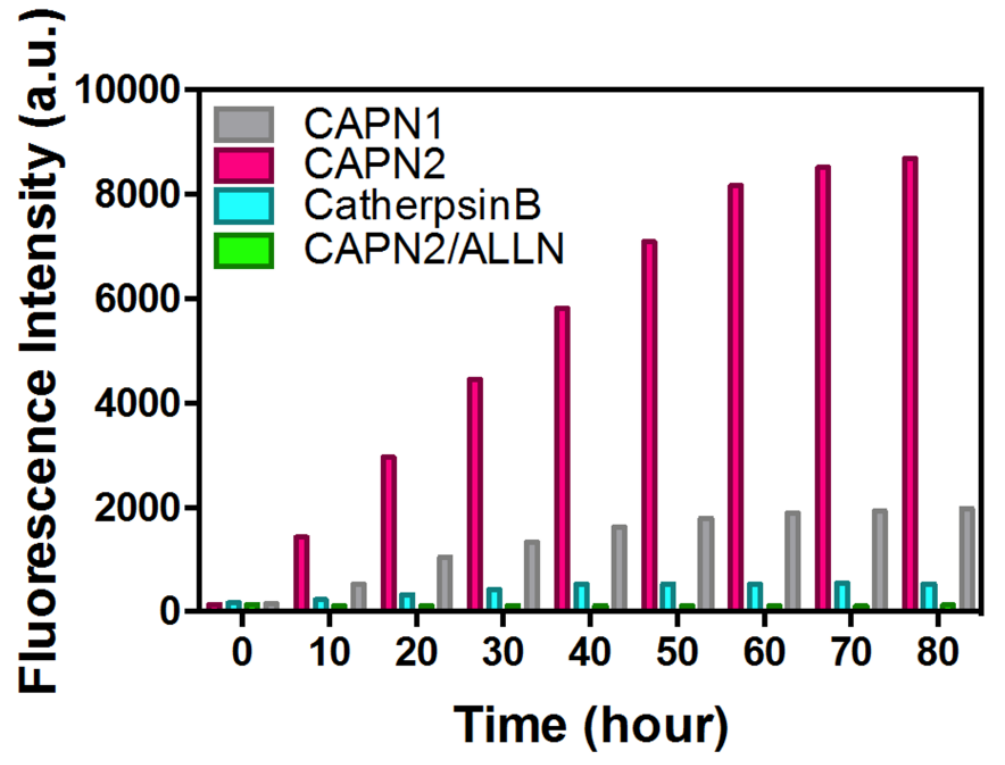

Figure S1. Characterization of CAPN2 peptide sensor. A. MALDI-TOF of CAPN2 peptide sensor. B. Recovered fluorescence signal of CAPN2 peptide sensor under the various enzyme conditions for 60 $\min$ at $37{ }^{\circ} \mathrm{C}$. 
A

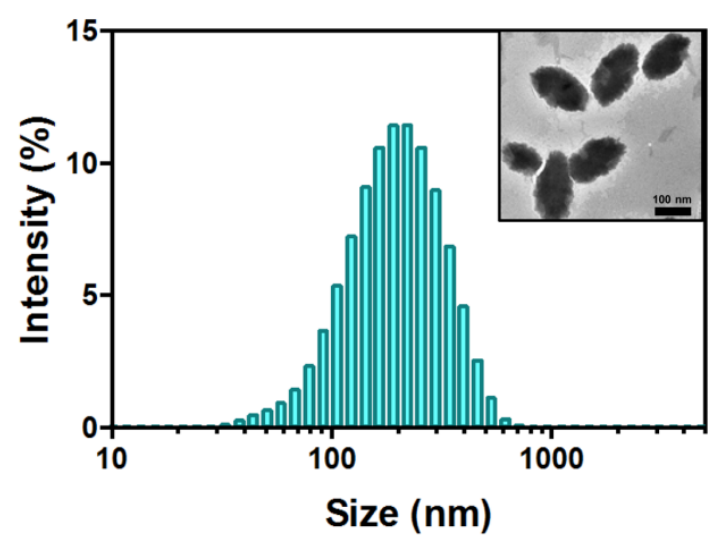

C

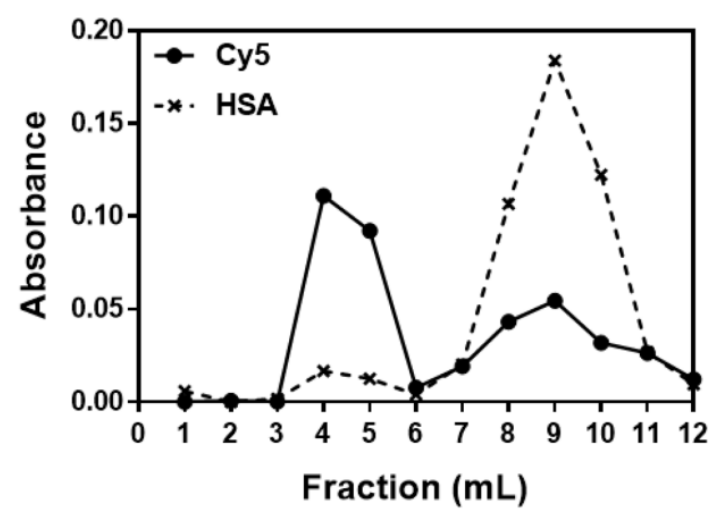

B

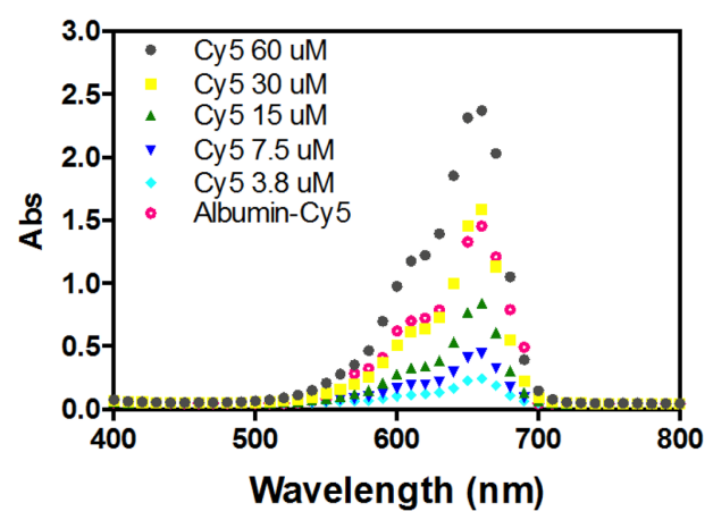

D

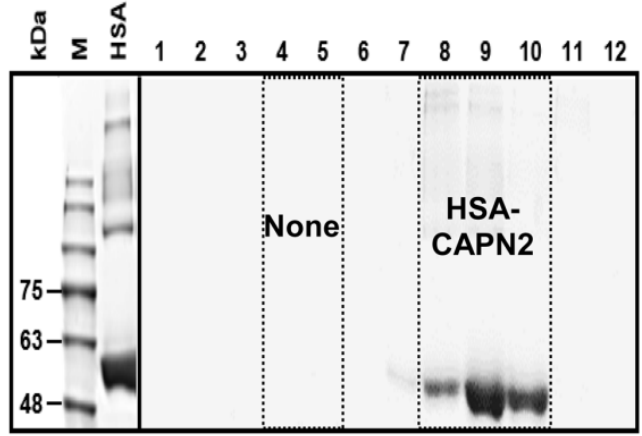

Figure S2. Characterization of HSA-Cy5 and HSA-CAPN2. A. Size distribution of HSA-Cy5 in phosphate-buffered saline ( $\mathrm{pH}$ 7.4). Transmission electron microscope image of HSA-Cy5 (insert). B. UV absorbance of HSA-Cy5 $(7.5 \mu \mathrm{M})$ and six different concentration of Cy5 $(3.8,7.5,15,30$ and 60 $\mu \mathrm{M})$. C. Size exclusion chromatography (SEC) profile of HSA-CAPN2. D. SDS-PAGE analysis of SEC fraction of HSA-CAPN2. 
A

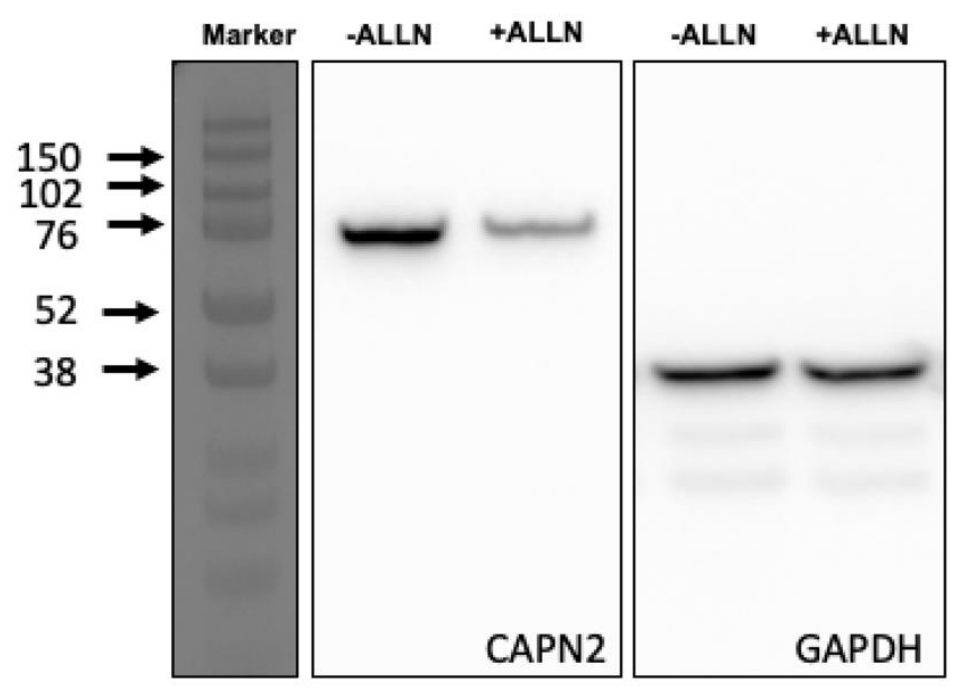

B

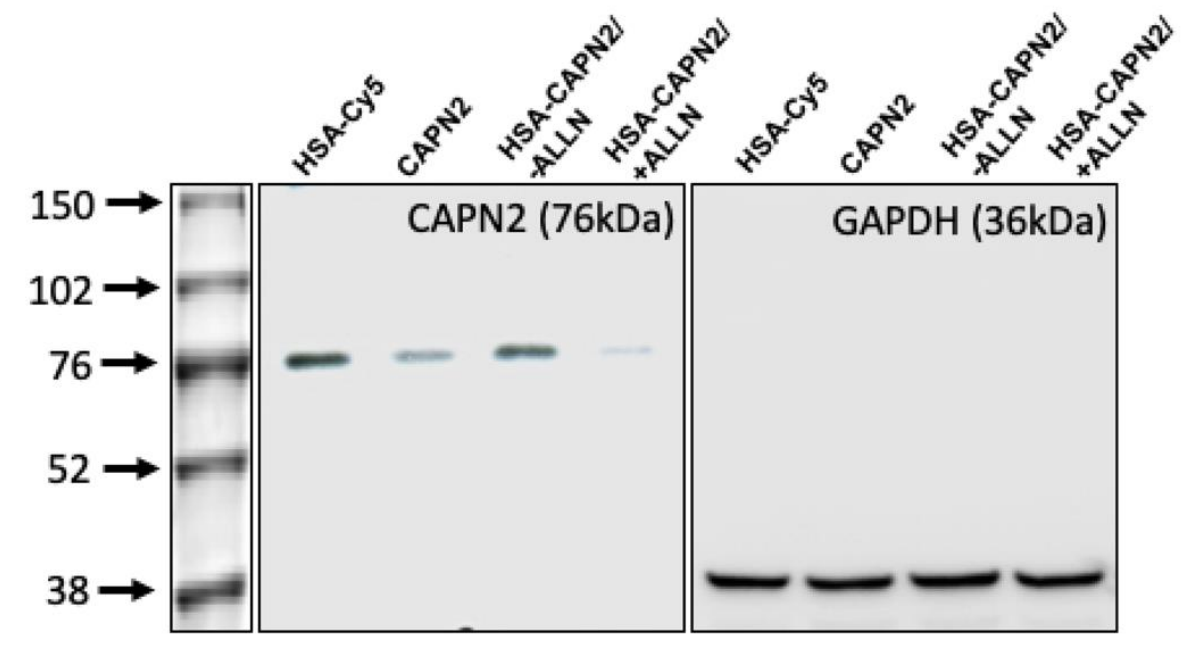

Figure S3. Full length blot images of cell and tissues CAPN2. A. CAPN2 protein expression with or without ALLN treatment in A549-Luc cells. B. CAPN2 protein expression in tumor tissues. $(10 \mu \mathrm{g} /$ lane) 
A

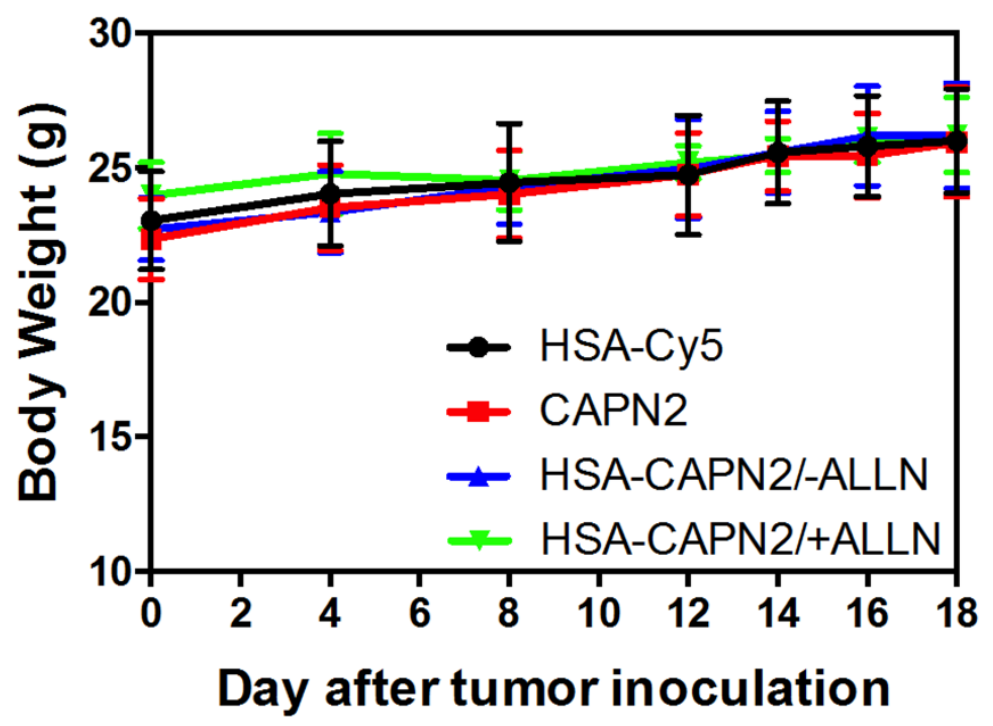

B

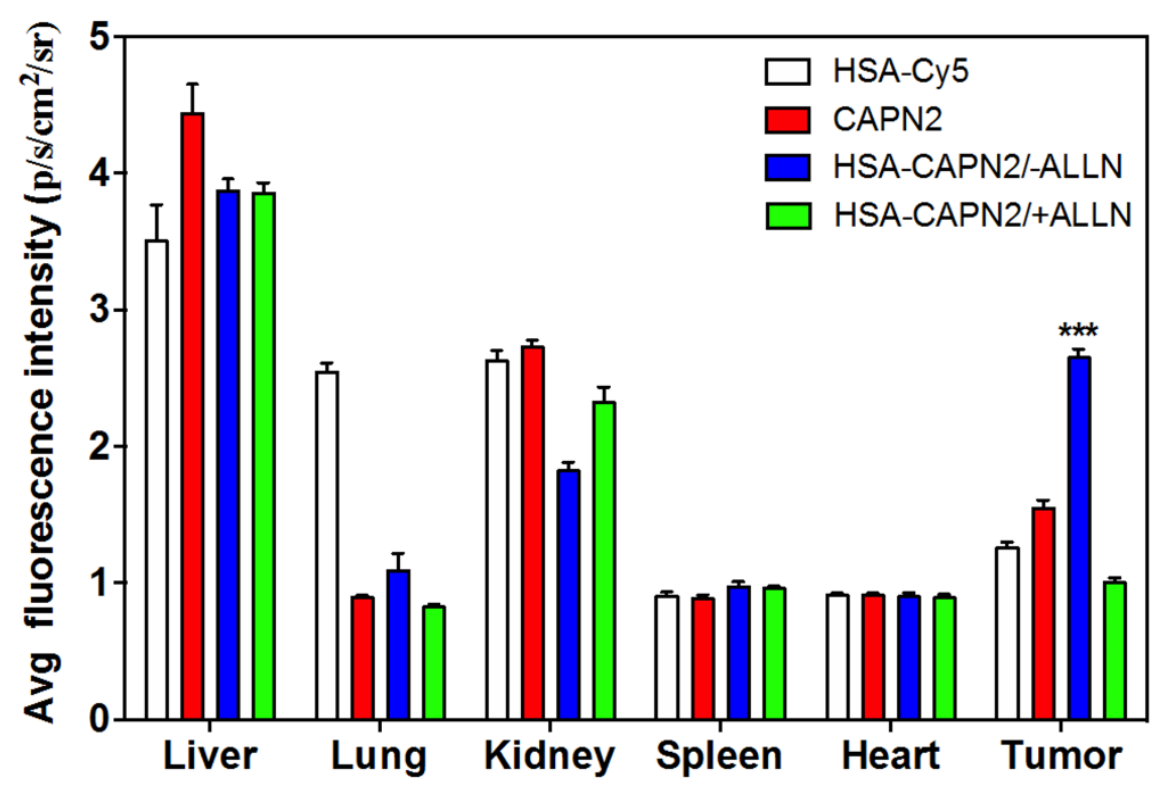

Figure S4. In vivo bodyweight and ex vivo organ fluorescence intensity. A. Bodyweight of mice after tumor inoculation. B. Quantification of main organ fluorescence intensity. Results are pres ented as mean \pm SEM values $(n=3)$. ${ }^{* *}$ indicate $p<0.001$. 

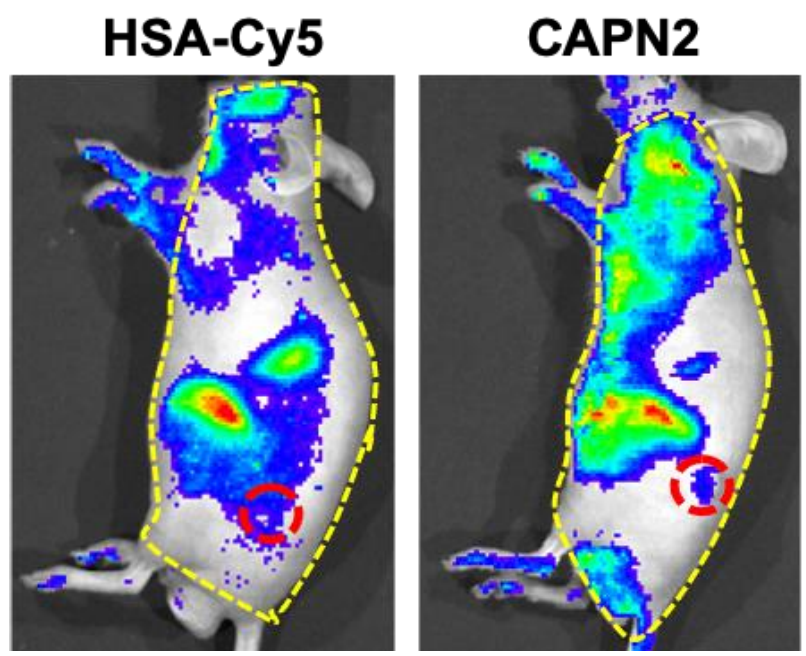

\section{HSA-CAPN2}

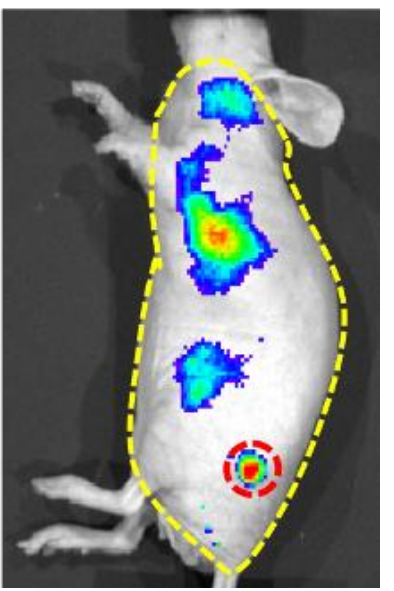

Figure S5. Estimating tumor-targeting efficacy. The mice whole body (yellow line) and tumor (red lin e) regions were measured $(n=3)$. 